\title{
A new genomic island
}

\begin{abstract}
A large collection of human genomes from Sardinia is reported in three linked papers that implicate new genetic variants in the regulation of height, blood lipids, inflammatory markers and hemoglobin levels. These analyses provide new insight into disease susceptibility and evolution in isolated human populations and illuminate the genetics of complex phenotypes.
\end{abstract}

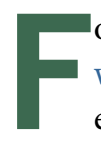
ollowing the pioneering 1000 Genomes Project (http:// www.nature.com/1000Genomes/), there have been several large-scale efforts to sequence and analyze individual whole genomes in human populations, including populations from the Netherlands (Nat. Genet. 46, 818-825, 2014), Iceland (http://www.nature.com/ng/focus/icelanders/) and the UK (Nature 526, 82-90, 2015).

An international consortium named the SardiNIA Project (https://sardinia.irp.nia.nih.gov/), led by Francesco Cucca, Gonçalo Abecasis, David Schlessinger and John Novembre, reports $\sim 17.6$ million genetic variants from whole-genome sequencing of 2,120 Sardinians; $22 \%$ of these variants were not found in any previous genomic data sets, and $\sim 76,000$ common variants (frequency $>5 \%$ ) are rare in other populations (frequency $<0.5 \%, 1000$ Genomes Project). In addition to this sequencing, the consortium also genotyped 6,602 Sardinians using array technology.

In Sidore et al. (page 1272), the team investigated the impact of variants on circulating lipid levels and five inflammatory biomarkers, for which they found 14 signals (two new loci) and 19 signals (two new loci), respectively. Importantly, some of these signals were detected because of highly accurate imputation that was based on the Sardinian sequencing data; these would not have been identified if the analyses had used 1000 Genomes Project data. Two examples of these signals are an $H B B$ mutation $\left(\beta^{0} 39\right.$, encoding p.Gln $\left.40^{*}\right)$ that associates with reduced cholesterol levels and an APOA5 missense variant (encoding p.Arg282Ser) that associates with a reduction in triglyceride levels.

In Danjou et al. (page 1264), the authors looked for associations with levels of fetal hemoglobin $(\mathrm{HbF})$ and adult hemoglobins A1 (HbA1) and A2 (HbA2) and identified 23 associations at ten loci, five of which are novel (MPHOSPH9, PLTP-PCIF1, ZFPM1 (FOG1), NFIX and CCND3). Apart from Spain, the NFIX variant seems to be absent in Europeans.
These findings have potential clinical value because these loci could act as modifiers of disease severity in patients with hemoglobinopathies.

Finally, in Zoledziewska et al. (page 1352), the authors studied the genetics of adult height variation in Sardinians, who constitute one of the shortest populations in Europe. They found two common variants with large effects: a nonsense mutation in $G H R$, a gene previously associated with Laron syndrome, and a mutation in the vicinity of KCNQ1. Interestingly, the latter variant is in an imprinted region and shows an association with reduced height when maternally inherited. Height is known to be a highly polygenic phenotype, and there are over 700 reported variants associated with height variation. The authors found that variants previously associated with reduced stature are more frequent than expected by genetic drift. These results suggest that short stature was selected for in Sardinia and seem to illustrate the so-called 'island effect' where the size of large mammals is reduced on islands.

The Sardinian studies reiterate the importance of studying isolated populations (see page 1224). These populations are often less genetically diverse and can be invaluable in characterizing the genetic underpinnings of monogenic and polygenic traits and diseases. However, analyzing these founder population data sets also poses a challenge: because some of the variants are very rare or nonexistent in other populations, it is not easy to replicate the associated signals in independent, unrelated cohorts. Careful examination of such variants in both in vitro and in vivo followup studies will help to clarify their biological roles.

The SardiNIA Project is part of a growing collection of largescale human genome sequencing endeavors. Analyses of these and future data sets will no doubt deepen our understanding of human biology and history and will hopefully contribute to the ongoing improvement of human health.

Sardinia is now a prominent island on the global genomic map. 